\begin{tabular}{|c|l|}
\hline Title & UItrafast carrier diffusion in gallium arsenide probed with picosecond acoustic pul ses \\
\hline Author(s) & Wright, O. B.; Perrin, B.; Matsuda, O.; Gusev, V. E. \\
\hline Citation & $\begin{array}{l}\text { PHY SICAL REVIEW B, 64, 081202(R) } \\
\text { https://doi.org/L0.1103/PhysRevB.64.081202 }\end{array}$ \\
\hline Issue Date & 2001 \\
\hline Doc URL & http://hdl.handle.net/2115/5797 \\
\hline Rights & Copyright $\odot 2001$ American Physical Society \\
\hline Type & article \\
\hline File Information & PRB64.pdf \\
\hline
\end{tabular}

Instructions for use 


\title{
Ultrafast carrier diffusion in gallium arsenide probed with picosecond acoustic pulses
}

\author{
O. B. Wright,${ }^{1}$ B. Perrin, ${ }^{2}$ O. Matsuda,${ }^{1}$ and V. E. Gusev ${ }^{3}$ \\ ${ }^{1}$ Department of Applied Physics, Faculty of Engineering, Hokkaido University, Sapporo 060-8628, Japan \\ ${ }^{2}$ Laboratoire des Milieux Désordonnés et Hétérogènes, Université Pierre et Marie Curie, UMR 7603 CNRS, \\ 4 Place Jussieu, 75252 Paris, France \\ ${ }^{3}$ Laboratoire de Physique de l'Etat Condensé, UMR-CNRS 6087, Faculté des Sciences, Université du Maine,
} Av. O. Messiaen, 72085 Le Mans, France

(Received 7 May 2001; published 2 August 2001)

\begin{abstract}
We describe an experimental investigation of the generation and detection of picosecond acoustic-phonon pulses in a thin slab of GaAs using ultrashort optical pulses. Comparison of the optical phase variation with a simple theory for ambipolar diffusion indicates that carrier diffusion has a significant effect on the shape of the phonon pulses generated. The phonon pulse duration is measured to be $\sim 25 \mathrm{ps}$, four times longer than that expected from optical-absorption considerations alone, indicating that hot carriers penetrate more than $100 \mathrm{~nm}$ into the sample during the phonon pulse generation process.
\end{abstract}

DOI: 10.1103/PhysRevB.64.081202

PACS number(s): 73.50. $-\mathrm{h}, 43.35 .+\mathrm{d}, 62.65 .+\mathrm{k}$

Ultrafast carrier diffusion in semiconductors has been studied by a variety of experimental techniques. Ultrashort pulse optical pump and probe methods based on the measurement of optical reflectivity, electro-optic sampling or luminescence, including near-field methods, have been applied to the measurement of diffusion of hot carriers in bulk semiconductors and quantum nanostructures in the lateral or through-thickness directions. ${ }^{1-4}$ The detection principle in this case involves the coupling of the carriers to the electric field of the optical wave. Optical pump and probe methods can also exploit the coupling of the carriers to strain, and can be used to monitor carrier diffusion from the shape of the acoustic-phonon pulses generated, a technique that involves the pulse-echo methods of laser picosecond acoustics. ${ }^{5,6}$ The penetration of hot carriers perpendicular to metal surfaces when excited with an ultrashort optical pulse has been shown to broaden the phonon pulses generated. ${ }^{7}$ Laser acoustics studies with nanosecond or sub-nanosecond temporal resolution in crystalline $\mathrm{Ge}$ and $\mathrm{Cd}_{x} \mathrm{~S}_{1-x}$ Se have demonstrated that carrier diffusion similarly affects acoustic generation in semiconductors. ${ }^{8,9}$ However, there have been no studies of the effect of carrier diffusion on acoustic generation in semiconductors with picosecond time resolution. This is unfortunate in view of the pressing need for such studies to support industrial development in quantitative nondestructive evaluation of integrated circuits or semiconductor nanostructures. There are also possible applications in the field of $\mathrm{GHz}-\mathrm{THz}$ acousto-optic modulation in ultrahigh speed semiconductor devices. Moreover, probing carrier diffusion on ultrashort timescales from acoustic measurements provides an interesting perspective on the time- and space-dependent nonequilibrium carrier distribution, because it allows depth profiling of the carrier penetration into the bulk. ${ }^{7}$ Despite this fundamental and practical interest previous laser picosecond acoustics experiments with semiconductor thin films involved complex GaAs multilayer geometries, and were not designed for the investigation of carrier diffusion. ${ }^{10-13}$

There are several challenging experimental problems related to such studies of carrier diffusion in crystalline semiconductors on picosecond timescales. First one must over- come the dual requirement for a sample of micron order or less in thickness (to avoid significant acoustic propagation distortion) and for an acoustically mismatched substrate (to allow significant echo amplitudes to be obtained). Molecularbeam epitaxy or related techniques for crystalline GaAs film growth, for example, are not ideal for this purpose because the lattice-matched substrate will invariably have a very similar acoustic impedance. Another problem is connected with the signal analysis of the acoustic echoes. The optical reflectivity changes resulting from the detection of acousticstrain pulses in an opaque material depend on the photoelastic effect, and because the optical penetration into the sample is usually of the same order or greater than the acoustic wavelength (typically a few tens of nanometers) these changes are related to the acoustic strain in a complex way. It is therefore difficult, in general, to resolve the strain pulse shape by monitoring only the optical reflectivity signal in laser picosecond acoustics.

In this study we have prepared a thin slab of $n$-doped GaAs bonded to an acoustically mismatched substrate to overcome the first set of problems. In addition, by using a two-color pump and probe technique combined with an interferometric dual-signal detection scheme in which both the optical reflectance and phase changes are monitored, we have succeeded in probing in a crystalline semiconductor sample the effect of hot carrier diffusion on the acousticstrain pulse shape generated by sub-picosecond laser pulses. The results suggest that the frequency of the generated acoustic pulses can be controlled by varying the doping concentration.

We use the photon excitation energy $E=3.3 \mathrm{eV}$ (wavelength $375 \mathrm{~nm}$ ) in order to localize the optical absorption to within $\sim 10 \mathrm{~nm}$ of the surface. Frequency doubled optical pump pulses of duration $\sim 200 \mathrm{fs}$ and average beam power 3-6 $\mathrm{mW}$ are derived from a mode-locked Ti:sapphire laser with an $82-\mathrm{MHz}$ repetition rate. These excite phonon pulses in a $2.6 \mu \mathrm{m}$ slab of (100) $n$-doped GaAs (Si doped to 1.3 $\times 10^{18} \mathrm{~cm}^{3}$ ). To make the sample a piece of a GaAs wafer was attached by a $0.75 \mu \mathrm{m}$ layer of ultraviolet-cured adhesive to a glass substrate and then mechanically polished. De- 


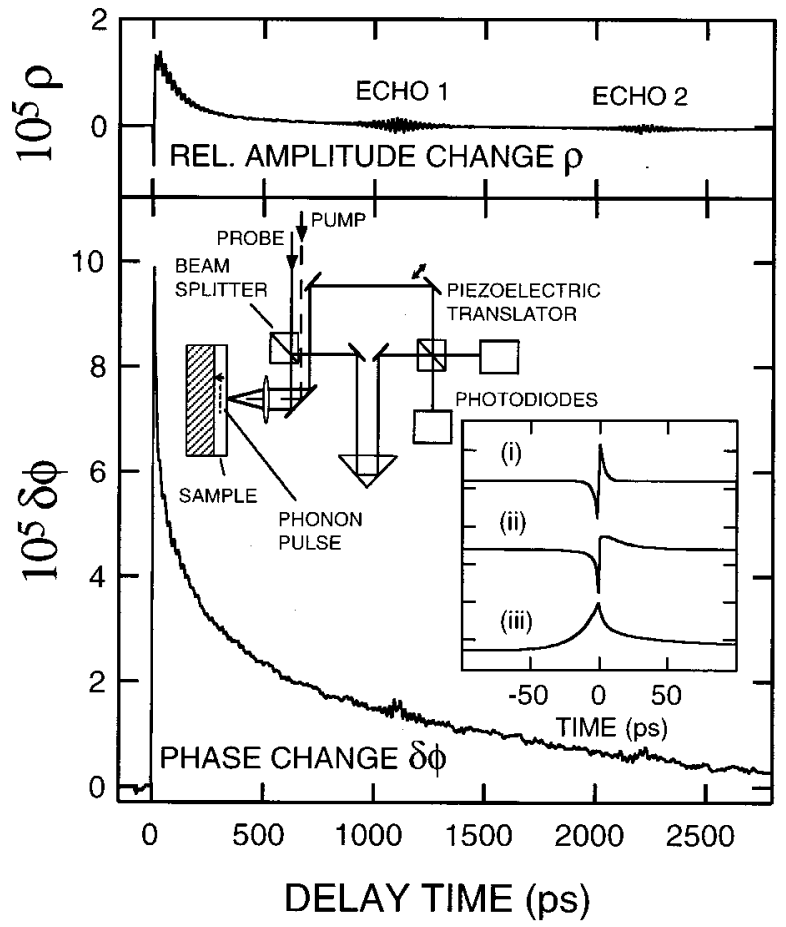

FIG. 1. Changes in $\rho$ (upper graph) and $\delta \phi$ (lower graph), the real and imaginary parts of the relative variation in complex reflectance $\delta r / r$, respectively, as a function of delay time for a pump fluence of $0.016 \mathrm{~m} \mathrm{~J} \mathrm{~cm}^{-2}$. The experimental configuration is also shown. The inset shows theoretical predictions: (i) the strain pulse temporal variation for $D=0$; (ii) the strain pulse temporal variation for $D / \nu \zeta=5$; (iii) the temporal variation of the particle displacement corresponding to (ii). Graphs (i) and (ii) have the same vertical scale. The experimental data were taken with 9 and 2 scans of the delay line respectively, each scan taking $100 \mathrm{~s}$.

layed infrared probe optical pulses of photon energy $1.65 \mathrm{eV}$ (wavelength $\lambda=750 \mathrm{~nm}$ ) of similar optical pulse duration derived from the same laser are used to detect transient changes in reflectance and phase in a Mach-Zehnder interferometer arrangement shown in Fig. 1. ${ }^{13}$ Both pump and probe beams were focused to the same $\sim 35 \mu \mathrm{m}$ diameter spot at $\sim 10^{\circ}$ incidence. We monitor $\rho=\delta R / 2 R$ and optical phase $\delta \phi$, where $R$ is the reflectivity. The phase change $\delta \phi$ is obtained using the difference between the signals from the photodiodes. ${ }^{13}$ The relative amplitude change $\rho$ and phase change $\delta \phi$ are related to the complex reflectance $r$ by $\delta r / r$ $=\rho+i \delta \phi$. $^{13,14}$

Two acoustic echoes can be seen in the data for $\rho$ and $\delta \phi$ in Fig. 1, for an incident pump fluence $F \sim 0.016 \mathrm{~m} \mathrm{~J} \mathrm{~cm}^{-2}$. These echoes are superimposed on a slow background variation caused by thermal diffusion. The difference between the first and second echo amplitudes is consistent with the acoustic reflection coefficient $r_{\mathrm{ac}} \approx-0.6$ at the GaAs-adhesive boundary (as expected from estimates based on literature values of acoustic impedances). The echo shapes were independent of fluence $F$ over the range $0.008-0.016 \mathrm{~m} \mathrm{~J} \mathrm{~cm}^{-2}$ studied. The oscillations, at frequency $2 n \nu / \lambda \approx 47 \mathrm{GHz}$ ( $n$ the probe refractive index and $v$ the longitudinal sound velocity) arise because of the photoelastic effect. ${ }^{5}$ The echo duration is $\sim 2 \zeta_{0} / v \approx 250 \mathrm{ps}$, where $\zeta_{0} \approx 600 \mathrm{~nm}$ is the probe optical- absorption depth. The phase change $\delta \phi(t)$ also includes a contribution equal to $-4 \pi \delta z / \lambda$, where $\delta z \sim 1 \mathrm{pm}$ is the outward displacement of the free surface. ${ }^{6,13,14}$ This contribution corresponds to the raised portion in the center of the echoes in $\delta \phi$, indicating that the phonon pulse duration is $\sim 20-40$ ps (with a frequency $\sim 15 \mathrm{GHz}$ ). In contrast, the variation $\rho(t)$, independent of the surface displacement, is not sensitive to the exact duration of the strain pulse. Although the echo duration is broadened by the probe optical penetration, this turns out to be an advantage in the signal analysis because it allows a clear separation of the effects of the photoelastic effect and the transient surface displacement.

Strain generation depends on the optical excitation of electron-hole $(e-h)$ pairs, a plasma of density $N$ with excess energy $E-E_{g}\left(E_{g}=1.43 \mathrm{eV}\right.$ is the lowest direct band gap at room temperature). While diffusing these hot carriers relax within a few picoseconds to the region of the conductionband edge (the $\Gamma$ point), releasing this excess as thermal energy. Subsequent evolution depends on the delayed heating through bulk and surface recombination and on further carrier and thermal diffusion. At low plasma densities $\left(\sim 10^{18} \mathrm{~cm}^{-3}\right.$ here $)$ and for timescales $\sim 10$ ps over which the acoustic-phonon pulse generation occurs, ${ }^{15}$

$$
\frac{\partial N}{\partial t}=D \frac{\partial^{2} N}{\partial z^{2}}+\frac{\left(1-R_{0}\right)}{\zeta C} I f(t) \exp (-z / \zeta),
$$

where, at the surface, $D \partial N / \partial z=S N$. Here $D$ is the ambipolar diffusion coefficient, $C$ the volume specific heat, $R_{0}$ the pump reflectivity, $S$ the surface recombination velocity, and If $(t)$ the intensity variation of the optical pulse $[f(t)$ is normalized]. At the low fluences used, the dependence of constants (such as $D$ or $\zeta$ ) on $N$ or temperature $T$ can be neglected. In GaAs the surface recombination velocity is typically subsonic, ${ }^{2}$ and should have a negligible influence on sound generation, so we take $\partial N / \partial z=0$ at the surface $(z=0)$. This assumption is examined later in this paper. Bulk recombination can be ignored for the timescales appropriate here.

In cubic semiconductors the fast relaxation of the plasma to the conduction-band edge sets up an initial isotropic stress given by the sum of the thermal stress and the electronic stress arising from the hydrostatic deformation potential $\propto \partial E_{g} / \partial p$. For the nonpiezoelectrically active (100) direction in GaAs,

$$
\sigma_{11}=\sigma_{22}=\sigma_{33}=-B \frac{\partial E_{g}}{\partial p} N-3 B \beta\left(T-T_{0}\right),
$$

where $B$ is the bulk modulus, $\beta$ is the linear thermal expansion coefficient, and where $N, T$, and $\sigma_{i i}$ in this equation refer to a time just after carrier relaxation to the conductionband edge. On timescales that are short compared to the sound propagation time across the optical spot diameter $(\sim 2-5 \mathrm{~ns})$, a longitudinal stress pulse is created traveling perpendicularly to the surface:

$$
\frac{\partial \sigma_{33}}{\partial z}=\rho_{0} \frac{\partial^{2} u}{\partial t^{2}}=\rho_{0} \nu^{2} \frac{\partial^{2} u}{\partial z^{2}}+B \frac{\partial E_{g}}{\partial p} \frac{\partial N}{\partial z}+3 B \beta \frac{\partial T}{\partial z},
$$


where $\rho_{0}$ is the density, $u=u(z, t)$ is the ultrasonic displacement, and $\sigma_{33}(z, t)$ is the longitudinal stress ( $=0$ at the surface). A knowledge of $N(z, t)$ and $T(z, t)$ allows the acoustic strain pulse $\eta_{33}(z, t)=\partial u / \partial z$ to be calculated. $T(z, t)$ is governed by thermal diffusion with source terms due to the spatiotemporal variation of the carrier energy. In contrast to the case for $\mathrm{Si}$ and $\mathrm{Ge},{ }^{8,15}$ the electronic and thermal strains have the same sign in GaAs, and their ratio is approximately $\left(\partial E_{g} / \partial p\right) C /\left[3 \beta\left(E-E_{g}\right)\right] \approx 5.8 \quad\left[\right.$ where $\quad \partial E_{g} / \partial p \approx 9$ $\times 10^{-11} \mathrm{eV} / \mathrm{Pa}$ (Ref. 17)]. It is therefore reasonable to neglect the thermal strain here. Carrier diffusion should broaden the acoustic strain pulse according to the value of $D / \nu \zeta$, where $\zeta$ is the optical-absorption depth for the pump light, in a way exactly analogous to the case for thermal diffusion in the absence of electronic strain. ${ }^{5}$ The analytical solution of Eqs. (1) and (2) for the strain pulse $\eta_{33}(z, t)$ $=\sigma_{33}(z, t) / \rho_{0} \nu^{2}$ in this case for the short optical pulse duration limit and when the strain pulse has traveled into the bulk of the solid is given by ${ }^{16}$

$$
\begin{aligned}
\frac{\eta_{33}(\theta)}{\eta_{0}}= & \frac{1}{1-M}\left[\frac{M}{M+1} e^{\theta / M}-\frac{1}{2} e^{\theta}\right] \quad \text { for } \theta<0, \\
= & \frac{1}{M+1}\left[\frac { M } { 1 - M } \left\{e^{\theta / M} \operatorname{erfc} \sqrt{\theta / M}\right.\right. \\
& \left.\left.-M e^{M \theta} \operatorname{erfc} \sqrt{M \theta}\right\}+\frac{1}{2} e^{-\theta}\right] \text { for } \theta>0,
\end{aligned}
$$

where $\theta=(\nu t-z) / \zeta, M=D / \nu \zeta$ and $\eta_{0}=B\left(\partial E_{g} / \partial p\right) F(1$ $\left.-R_{0}\right) /\left(E \zeta \rho_{0} \nu^{2}\right)$. Calculations based on this equation are shown in the inset of Fig. 1: the temporal forms of the strain pulse shape for (i) $D=0$ and (ii) $D / \nu \zeta=5$ are shown together with (iii) the displacement variation associated with the strain pulse for $D / \nu \zeta=5$. (This value of $D$ is found later.) For $D=0$ the strain pulse has a duration $\sim 2 \zeta / \nu \approx 6 \mathrm{ps}^{5,6}$ Carrier diffusion distorts the shape and leads to an increased duration. Once $\eta_{33}(z, t)$ is known, $\rho(t)$ and $\delta \phi(t)$ can be calculated from the known photoelastic constants and from $\delta z(t)=\int \eta_{33}(z, t) d z$ according to the equation ${ }^{5,14}$

$$
\begin{aligned}
\frac{\delta r}{r}= & \rho+i \delta \phi=-2 i k_{0} \delta z \\
& +\frac{4 i k_{0} \widetilde{n}}{\left(1-\widetilde{n}^{2}\right)} \frac{d \widetilde{n}}{d \eta} \int_{0}^{\infty} \eta_{33}(z, t) \exp \left(2 i k_{0} \widetilde{n} z\right) d z,
\end{aligned}
$$

where $\widetilde{n}=n+i \kappa$ is the probe refractive index and $k_{0}$ $=2 \pi / \lambda$ is the free space probe wave vector. In general, the strain field $\eta_{33}(z, t)$ contains components traveling in both the $+z$ and $-z$ directions when the strain pulse is being reflected from the surface.

To facilitate an accurate comparison of theory and experiment we have taken careful measurements of the first echo shape at $F \sim 0.008 \mathrm{~m} \mathrm{~J} \mathrm{~cm}^{2}$ using an optical delay line incorporating a shaker, ${ }^{18}$ as shown by the solid curves in Fig. 2 (with the background variation subtracted). Using only $D / \nu \zeta$ and a single arbitrary multiplicative scale factor as variable quantities, we obtain through simultaneous least-squares fitting to $\rho$ and $\delta \phi$ the optimum value $D / \nu \zeta=5$, corresponding

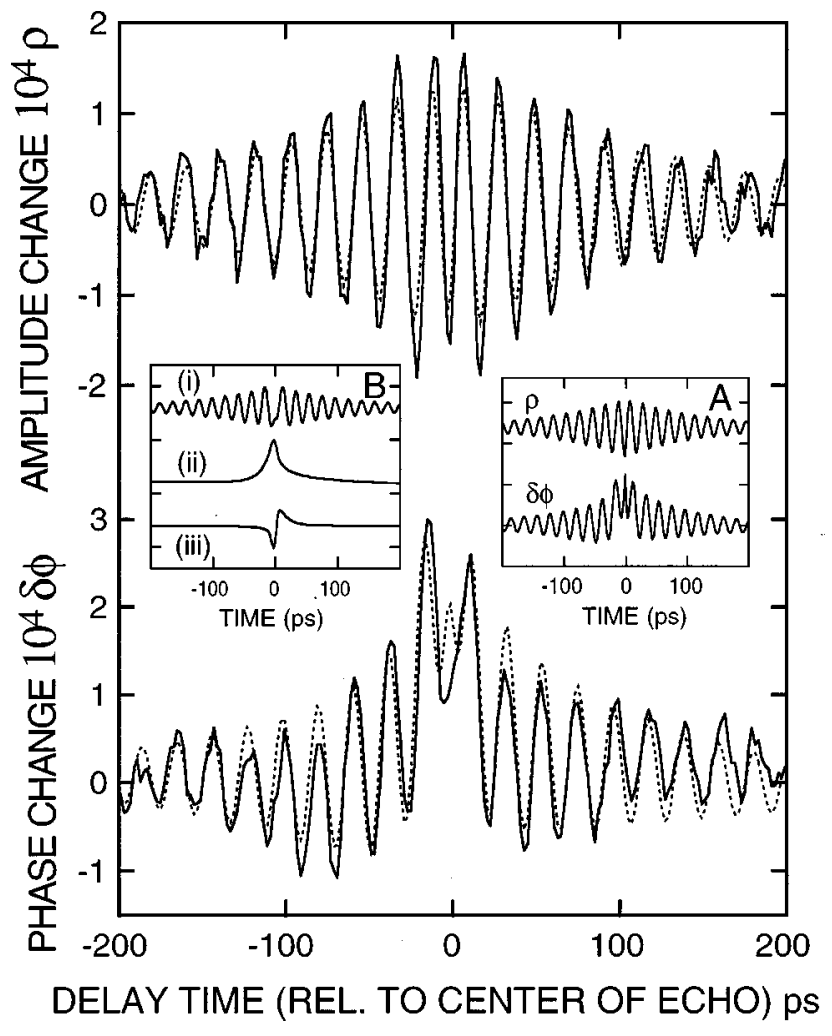

FIG. 2. Changes in $\rho$ (upper graph) and $\delta \phi$ (lower graph) as a function of delay time, for a pump fluence of $0.008 \mathrm{~m} \mathrm{~J} \mathrm{~cm}^{-2}$, for the first acoustic echo. The solid curves correspond to experimental data and the dotted curves to theoretical fits that include carrier diffusion $(D / \nu \zeta=5)$ and the effects of surface roughness and nonparallelism $(\tau=7 \mathrm{ps})$. The theoretical plots in inset A correspond to fits to $\rho$ and $\delta \phi$ that include carrier diffusion only $(D / \nu \zeta=5)$. The theoretical plots in inset $B$ show (i) the photoelastic contribution to $\delta \phi$, (ii) the surface displacement contribution to $\delta \phi$ [on the same scale as (i)], and (iii) the effective temporal variation of the strain, all corresponding to $D / \nu \zeta=5$ and $\tau=7 \mathrm{ps}$. The experimental data were taken with 2000 scans of the shaker.

to $D=3.3 \mathrm{~cm}^{2} \mathrm{~s}^{-1}$, using the known photoelastic constants $d n / d \eta=2.8$ and $d \kappa / d \eta=2.6$ (and with probe refractive index $n+i \kappa=3.76+0.1 i) .{ }^{19}$ The calculated $\rho(t)$ and $\delta \phi(t)$ curves are shown in the inset $A$ of Fig. 2. The agreement for $\rho(t)$ and the wings of the $\delta \phi(t)$ variation indicate that the $(d n / d \eta) /(d \kappa / d \eta)$ ratio is accurate. But, in contrast to experiment, the central portion of the predicted $\delta \phi(t)$ variation contains a sharp spike.

This sharp spike is not significantly changed if the finite duration of the optical pulses is included in the model. However, a smoothing effect would be expected if the surface(s) of the sample were rough or inclined as in a wedge. To check this possibility separate atomic-force microscope measurements were carried out. We measured a root-mean-square surface roughness of $5 \mathrm{~nm}$ for the top surface (much greater than the value $\sim 0.5 \mathrm{~nm}$ found for the as-manufactured bottom surface) and a wedge angle $\theta=0.33 \mathrm{mrad}$. We estimate that this results in the requirement for a convolution of $\rho(t)$ and $\delta \phi(t)$ with a Gaussian temporal smoothing function with $\tau_{1}=5$ ps FWHM (full width at half maximum) to in- 
clude the effect of surface roughness and with $\tau_{2}=3.5 \mathrm{ps}$ to include the effect of nonparallelism. ${ }^{20}$ Taken together we would expect a $\tau=\left(\tau_{1}^{2}+\tau_{2}^{2}\right)^{1 / 2} \approx 6$ ps FWHM Gaussian smoothing of the echo shape. We repeated the least-squares fitting with both $D$ and $\tau$ as variable quantities, and find the optimum values $D / \nu \zeta=5$ and $\tau=7 \mathrm{ps}$, in good agreement with the estimated value of $\tau$. The results are shown by the dotted curves in Fig. 2. The sum of the mean-square deviations was decreased by $25 \%$ compared with $\tau=0$ and the central peak in the theoretical fit to $\delta \phi(t)$ is much reduced in height. Moreover, the predicted absolute magnitude is of the correct order. (For example, the echo $\rho$ in Fig. 2 is predicted, including the effect of the probe beam size, to have a maximum height of $\sim 2 \times 10^{-5}$ compared with $3 \times 10^{-5}$ observed.) Fits from three different sets of data all gave optimum values of $D / \nu \zeta$ in the range $D / \nu \zeta=5 \pm 1$, corresponding to hot carrier penetration to a distance $\nu \Delta t$ $\sim 120 \mathrm{~nm}$ into the solid, where $\Delta t \sim 25 \mathrm{ps}$ is the FWHM value of $\delta z(t)$. In the inset $B$ in Fig. 2 we also show (i) the photoelastic contribution to $\delta \phi$, (ii) the surface displacement contribution to $\delta \phi$, and (iii) the effective strain pulse shape for $D / \nu \zeta=5$ and $\tau=7$ ps.

The value of the ambipolar diffusivity $D$ required to fit the results is significantly less than the value $D \approx 12 \mathrm{~cm}^{2} \mathrm{~s}^{-1}$ for intrinsic GaAs for $N \leqslant 10^{18} \mathrm{~cm}^{-3}$. ${ }^{1}$ This value of $D$ would lead to a strain pulse duration twice that derived here. However, $D$ is smaller at finite donor densities: at our relatively low values of $N$,

$$
D=\frac{\mu_{e} D_{h}+\mu_{h} D_{e}}{\mu_{e}+\mu_{h}}=\frac{2 D_{h} D_{e}}{D_{e}+D_{h}} \approx 2 D_{h},
$$

where $D_{e}$ is the (majority) electron diffusivity and $D_{h}$ $\left(\ll D_{e}\right.$ ) is the (minority) hole diffusivity, $\mu_{e, h}$ is the mobility, and use has been made of the Einstein relations $\mu_{e, h}$ $=e D_{e, h} / k T$. For the present donor density, $2 D_{h}$ has been found in the range $3-12 \mathrm{~cm}^{2} \mathrm{~s}^{-1},{ }^{17}$ not inconsistent with our derived value. The model we used does not account for the effects of near-surface electric fields and the complex carrier dynamics owing to intervalley scattering. ${ }^{2}$ Taking either of these factors into account might affect the phonon pulse shape, although it is difficult to see how such short time processes could significantly affect a phonon pulse duration $\sim 25$ ps. We also expect corrections due to the effects of pulse broadening from frequency-dependent acoustic attenuation to be negligible for our frequencies and propagation distance. ${ }^{10}$ Furthermore, calculations of the pulse shape including surface recombination did not improve the fits or change $D .^{21}$

In conclusion, we have measured, to the best of our knowledge, for the first time in a crystalline semiconductor the effect of hot carrier diffusion on the acoustic-phonon pulse shape generated by subpicosecond laser pulses. The experimental acoustic strain pulse shapes in GaAs can be quantitatively understood on the basis of a model of ambipolar carrier diffusion into the bulk of the solid. The experimental conditions are well suited to probe the diffusion of low-density electron-hole plasmas on sub-picosecond and picosecond timescales and on length scales $\sim 10-100 \mathrm{~nm}$, and further measurements with different doping should show a phonon pulse duration dependent on doping concentration. In the future it would be interesting to probe the role of the transient populations of different regions in $\mathbf{k}$ space with measurements at different optical wavelengths.
${ }^{1}$ R. Ziebold, T. Witte, M. Hubner, and R. G. Ulbrich, Phys. Rev. B 61, 16610 (2000).

${ }^{2}$ T. Dekorsy, T. Pfeifer, W. Kutt, and H. Kurz, Phys. Rev. B 47, 3842 (1993); Y. Rosenwaks, B. R. Thacker, R. K. Ahrenkiel, A. J. Nozik, and I. Yavneh, ibid. 50, 1746 (1994).

${ }^{3}$ M. Achermann, B. A. Nechay, F. Morier-Genoud, A. Schertel, U. Siegner, and U. Keller, Phys. Rev. B 60, 2101 (1999).

${ }^{4}$ H. W. Yoon, D. R. Wake, J. P. Wolfe, and H. Morkoc, Phys. Rev. B 46, 13461 (1992).

${ }^{5}$ C. Thomsen, H. T. Grahn, H. J. Maris, and J. Tauc, Phys. Rev. B 34, 4129 (1986).

${ }^{6}$ O. B. Wright and K. Kawashima, Phys. Rev. Lett. 69, 1668 (1992).

${ }^{7}$ O. B. Wright, Phys. Rev. B 49, 9985 (1994).

${ }^{8}$ N. V. Chigarev, D. Yu. Paraschuk, X. Y. Pan, and V. E. Gusev, Phys. Rev. B 61, 15837 (2000).

${ }^{9}$ W. Gao, V. Gusev, C. Glorieux, J. Thoen, and B. Borghs, Opt. Commun. 143, 19 (1997).

${ }^{10}$ W. Chen, H. J. Maris, Z. R. Wasilewski, and S. Tamura, Philos. Mag. B 70, 687 (1994).

${ }^{11}$ K. Mizoguchi, M. Hase, S. Nakashima, and M. Nakayama, Phys. Rev. B 60, 8262 (1999).

${ }^{12}$ A. Bartels, T. Dekorsy, H. Kurz, and K. Kohler, Phys. Rev. Lett. 82, 1044 (1999).
${ }^{13}$ B. Perrin, B. Bonello, J. C. Jeannet, and E. Romatet, Prog. Nat. Sci. S6, 444 (1996).

${ }^{14}$ D. H. Hurley and O. B. Wright, Opt. Lett. 24, 1305 (1999).

${ }^{15}$ O. B. Wright and V. E. Gusev, Appl. Phys. Lett. 66, 1192 (1995).

${ }^{16}$ V. E. Gusev, Acust. Acta Acust. 82, S37 (1996).

${ }^{17}$ S. Adachi, Gallium Arsenide and Related Materials (World Scientific, Singapore, 1994).

${ }^{18}$ The echo heights were proportional to the pump power, but varied slightly between measurements owing to changes in optical alignment.

${ }^{19}$ These were calculated by converting the photoelastic constants $P$ reported by P. Etchegoin et al., Phys. Rev. B 46, 15139 (1992) to those relevant here: $d n / d \eta+i d k / d \eta=\left[P_{11} c_{11}\right.$ $\left.+2 P_{12} c_{12}\right] / 2(n+i k)$, where $c_{11}$ and $c_{12}$ are elastic constants taken from Ref. 17.

${ }^{20} \mathrm{We}$ assume anticorrelation between the spatial profile of the wavefront of the acoustic pulse and the profile of the surface roughness when the acoustic pulse returns to the surface, leading to $\tau_{1}=4 \sigma(2 \ln 2)^{1 / 2} / \nu$, where $\sigma$ is the mean-square surface roughness. We calculate $\tau_{2}$ from $\tau_{2}=\sqrt{2} w \theta / \nu$, where $w$ $\approx 35 \mu \mathrm{m}$ is the FWHM optical spot diameter of the pump and probe.

${ }^{21}$ Values of $S / \nu$ up to $\sim 0.2$ gave reasonable fits. 